\title{
A study of the biochemical profile and its correlation with Alcohol Dependence: a hospital based study
}

\author{
Khushboo Dewani ${ }^{1}$, Narayan Mutalik ${ }^{2}$ \\ ${ }^{1}$ Senior Resident, Spandana Nursing Home, Bangalore, Karnataka. \\ ${ }^{2}$ Associate Professor, Department of Psychiatry, S.N.Medical College, Bagalkot. \\ Corresponding author: Narayan R. Mutalik \\ Email - narayanmutalik@gmail.com
}

\begin{abstract}
Background: The aim of the research was to study the biochemical profile of the male patients diagnosed with alcohol dependence syndrome and its correlation with the severity of dependence.

Methodology: A descriptive study comprising of 60 patients admitted in the psychiatry de-addiction clinic at HSK hospital from 1st July 2017 to 31st December 2017 was done after taking institutional ethical committee clearance. A semi-structured proforma was used to determine the socio-demographic details like education, occupation, socio-economic status etc. the severity of dependence was determined using Severity of Alcohol Dependence Questionnaire (SADQ) scale and basic biochemical tests were performed. Descriptive analysis using an appropriate statistical test was done.

Results: The mean age at presentation was 37.9 years. Out of 60 patients, 21 (35\%) had mild dependence, $24(40 \%)$ had moderate dependence and 15 (25\%) had severe dependence. Hepatic enzymes AST and ALT were raised in $76.67 \%$ and $66.67 \%$ patients respectively. AST to ALT ratio was above 2 in $11.67 \%$ patients. There was a significant correlation between total SADQ scores and Alanine Transaminase $(\rho=0.281, \mathrm{P}=$ $0.03, \mathrm{n}=60)$.

Conclusion: The biochemical tests were deranged in most of the patients with alcohol dependence. Certain markers can be developed based on their significant association with the dependence levels, thus, helping in early diagnosis and prevention of alcohol dependence syndrome.
\end{abstract}

Keywords: Alcohol dependence, S-aspartate-aminotransferase, S-alanine-aminotransferase, alcohol, biochemical profile.

\section{(Paper received $-20^{\text {th }}$ September 2020 , Peer review completed $-15^{\text {th }}$ October 2020$)$}

(Accepted $-19^{\text {th }}$ October 2020)

\section{INTRODUCTION}

Substance abuse has become a global phenomenon greatly influenced by social, economic, political, and psychosocial factors. The phenomenon not only includes the use of licit substances like alcohol, tobacco but also illicit substances such as cannabis, opioids, etc. Alcohol has been consumed in India for centuries. The worldwide prevalence of alcohol use disorders among the individuals at the age of 15 years and above was estimated to range from $0 \%$ to $16 \%$ [1]. The prevalence of use of alcohol ranges from a low of $7 \%$ in Gujarat (officially under Prohibition) to $75 \%$ in the North-eastern state of Arunachal Pradesh [2].

There is a subgroup of alcohol consumers who drink excessively so as to develop physical tolerance, withdrawal, loss of control and salience; and are diagnosed with alcohol dependence. These individuals are at high risk for multiple system failures due to micronutrient deficiency and the toxic effects of alcohol. Hence, there is an exaggerated risk for morbidity and mortality.

Various self-reporting screening questionnaires and laboratory tests can be used to help in the diagnosis of alcoholism. Various biochemical markers for alcohol abuse are available, but none is $100 \%$ efficient. A screening test should be highly sensitive and specific and discriminate between safe social drinking and 
heavy, hazardous drinking. The marker should not be elevated by non-alcoholic liver disease and should be non-invasive (e.g. urine, saliva, breath or blood test). A marker that is used to detect relapse should be sensitive to any alcohol consumption above safe levels [3].

S-aspartate-aminotransferase (AST), and S-alanine-aminotransferase (ALT) are frequently used in general practice for assessing liver function in health screening. AST and ALT are often raised in patients who are alcoholics, a 40- percent increase in AST is 90- percent sensitive, and a 20-percent increase in ALT is 80percent sensitive for relapse, yet both have low specificity [4]. Serum AST can also arise from non-hepatic sites, particularly heart and muscle, and levels are increased in conditions such as myocardial infarction and skeletal muscle trauma. The AST: ALT ratio improves the test; a ratio $>1.5$ strongly suggests, and a ratio > 2.0 is almost indicative of, alcohol-induced damage to the liver. One study has shown the AST: ALT ratio to be the best in distinguishing between alcohol-induced and non-alcoholic liver disease [3]. Deficiency of pyridoxal-5'-phosphate, a necessary coenzyme for both aminotransferases, is common in alcoholic liver disease. This deficiency causes a decrease in hepatic ALT to a greater extent than AST [5].

Electrolyte abnormalities are common in chronic alcoholics. One study showed that mixed disturbances were present in $78 \%$ of patients with alcohol dependence [6]. A study found hyponatremia (72.8\%) was the most frequent electrolyte disturbance in patients with alcohol dependence followed by hypomagnesemia (29.8\%) and hypokalemia (28.9\%) [7]. Another feature of chronic alcoholic liver disease is progressive hypoalbuminemia, the decrease in serum albumin level is attributed to the nutritional status of the subjects. On the other hand, the albumin is a potential subject of the formation of adduct by acetaldehyde, an alcohol metabolite. This albumin or other protein adducts can stimulate the formation of immunoglobulins, thus causing a rise in serum globulin levels [8].

Screening is helpful in identifying subjects at risk particularly in the early phase of drinking. It helps in identifying both hazardous and harmful users. The severity of alcohol dependence instruments is useful in patients with significant misuse but may not be sensitive to low-level use [9]. The ideal screening tool should be easy to administer, short and accurate, and correlate with other more complex diagnostic tests. Questionnaires are ideal for screening and can identify up to $80 \%$ of alcoholics, but they rely on the patient's truthfulness and memory [10]. The questionnaire method has depended on the severity of Alcohol Dependence Questionnaire (SADQ).

The current study has explored the biochemical profile of individuals diagnosed with alcohol dependence syndrome. We have also tried to find the correlation between various biochemical markers and the severity of alcohol dependence.

\section{METHODOLOGY}

The study was conducted in the De-Addiction Clinic of the Department of Psychiatry at a private medical college in Bagalkot, Karnataka during the period from 1st July 2017 to 31st December 2017. All the patients fulfilling the inclusion and exclusion criteria during the study period were included in this study.

\section{Inclusion Criteria}

All in-patients admitted through the de-addiction clinic of the HSK Hospital with alcohol-related problems were potential candidates for this study and were enrolled in the study if they fulfil the following inclusion criteria:

1. Patient of age 18 years and above

2. A patient who fulfils criteria for dependence according to ICD-10.

3. Willing to give written informed consent.

\section{Exclusion Criteria}

Subjects with any of the following were not included in the study:

1. History of any major illness requiring intensive medical/surgical intervention.

2. Not giving informed consent.

The design and nature of the clinical study was explained to the patients. Informed consent was obtained. All patients were first examined by consultant Psychiatrist to confirm the diagnosis of alcohol dependence. Socio-demographic details like education, occupation, socio-economic status etc. were collected using a semi-structured proforma, the severity of dependence was determined using Severity of Alcohol Dependence 
Questionnaire (SADQ) scale and basic biochemical tests were performed.

SADQ is a 20-item questionnaire formulated by Edwards and Gross. The original SADQ is divided into five sections:

(i) physical withdrawal symptoms,

(ii) affective symptoms of withdrawal,

(iii) craving and withdrawal relief drinking,

(iv) typical daily consumption and

(v) reinstatement of withdrawal symptoms after a period of abstinence.

It has been validated in inpatient, outpatient, and community settings [11].

Statistical analysis was done using SPSS for Windows (SPSS software package, trial version 20).

\section{RESULTS}

A total of 60 patients were included in the study. The mean age at presentation was 37.9 years $(S D=9.27)$. Minimum age being 20 years and maximum being 65 years. Table 1 gives the summary of the sociodemographic profile of the patients. Out of 60 patients, 21 (35\%) had mild dependence, $24(40 \%)$ had moderate dependence and $15(25 \%)$ had severe dependence according to SADQ scores dependent patients, $8(53.33 \%)$ had hypokalemia.

Table 1: Socio-demographic data

\begin{tabular}{|c|c|}
\hline Socio-demographic Data & N (\%) \\
\hline No. of Participants & 60 \\
\hline \multicolumn{2}{|c|}{ Education } \\
\hline Age (average) at presentation \\
\hline No Formal Education & 47.9 years \\
\hline Primary & $5(8.67)$ \\
\hline Secondary & $21(35)$ \\
\hline Higher Secondary & $14(23.33)$ \\
\hline Graduate & $12(20)$ \\
\hline Postgraduate & $4(6.67)$ \\
\hline \multicolumn{2}{|c|}{ Marital Status } \\
\hline Single & $12(20)$ \\
\hline Married and living with spouse & $43(71.67)$ \\
\hline Separated & $5(8.33)$ \\
\hline \multicolumn{2}{|c|}{ Onation } \\
\hline Unemployed & $4(6.67)$ \\
\hline Unskilled/Farmer/Clerical & $26(43.33)$ \\
\hline Semi-skilled/Skilled work & $22(36.67)$ \\
\hline Professional/ Business & $8(13.33)$ \\
\hline \multicolumn{2}{|c|}{ Socio- economic status } \\
\hline Above Poverty Line & $44(73.33)$ \\
\hline Below Poverty Line & $16(26.67)$ \\
\hline
\end{tabular}

Table 2: Biochemical profile of the subjects

\begin{tabular}{|c|c|c|}
\hline Variable & Median & Range, $\mathbf{9 5 \%}$ Cl \\
\hline FBS $(\mathrm{mg} / \mathrm{dL})$ & 100 & $68-158$ \\
\hline PPBS $(\mathrm{mg} / \mathrm{dL})$ & 140 & $94-264.7$ \\
\hline Urea $(\mathrm{mg} / \mathrm{dL})$ & 24 & $9-60$ \\
\hline Creatinine $(\mathrm{mg} / \mathrm{dL})$ & 0.90 & $0.5-1.3$ \\
\hline Total Protein & 6.35 & $4.4-8$ \\
\hline Albumin $(\mathrm{g} / \mathrm{dL})$ & 3.4 & $2.4-4.8$ \\
\hline Globulin $(\mathrm{g} / \mathrm{dL})$ & 2.95 & $1.2-4.4$ \\
\hline Total Bilirubin $(\mathrm{mg} / \mathrm{dL})$ & 0.95 & $0.3-2.8$ \\
\hline
\end{tabular}




\begin{tabular}{|c|c|c|}
\hline Direct Bilirubin (mg/dL) & 0.4 & $0.1-1.2$ \\
\hline Alkaline Phosphate (U/L) & 99.5 & $50-248$ \\
\hline AST (U/L) & 98 & $14-2000$ \\
\hline ALT (U/L) & 57.5 & $10-8300$ \\
\hline Sodium (meq/L) & 135 & $129-147$ \\
\hline Potassium (meq/L) & 3.6 & $2.9-5$ \\
\hline Chloride (meq/L) & 107 & $90-132$ \\
\hline
\end{tabular}

There Was A Significant Correlation Between Total SADQ Scores And Alanine Transaminase $(\mathrm{p}=0.281, \mathrm{p}=0.03, \mathrm{~N}=60)$. (Table 3$)$

Table 3: Correlation of Liver Function Test with Severity Of Alcohol Dependence

\begin{tabular}{|c|c|c|c|c|c|}
\hline Liver Function Test & \multicolumn{3}{|c|}{ SADQ } & Spearman Rho ( P) & P Value \\
\hline & Mild & Moderate & Severe & & \\
\hline \multicolumn{6}{|l|}{ ALT } \\
\hline Normal & 11 & 6 & 3 & \multirow{2}{*}{0.281} & \multirow{2}{*}{$0.03^{\mathrm{a}}$} \\
\hline High & 10 & 18 & 12 & & \\
\hline \multicolumn{6}{|l|}{ AST } \\
\hline Normal & 7 & 4 & 3 & \multirow{2}{*}{0.138} & \multirow{2}{*}{0.291} \\
\hline High & 14 & 20 & 12 & & \\
\hline \multicolumn{6}{|l|}{ Total Bilirubin } \\
\hline Normal & 12 & 18 & 6 & \multirow{2}{*}{0.094} & \multirow{2}{*}{0.473} \\
\hline High & 9 & 6 & 9 & & \\
\hline \multicolumn{6}{|l|}{ Alkaline Phosphate } \\
\hline Normal & 19 & 16 & 11 & \multirow{2}{*}{0.182} & \multirow{2}{*}{0.164} \\
\hline High & 2 & 8 & 4 & & \\
\hline
\end{tabular}

Note: ${ }^{a}$ Correlation is significant at the 0.05 level.

\section{DISCUSSION}

The present study was a hospital-based study and aimed at determining the relationship of liver enzymes with the severity of dependence in patients of alcohol dependence syndrome. The mean age at presentation was 37.9 years $(\mathrm{SD}=9.27)$. Mean age at presentation in previous studies with a similar design have ranged between 37 and 43 years [1,12]. A majority of individuals (73.3\%) belonged to the above poverty level group socio-economically. A study by Gedam and others [13] involving a rural population of central India found a majority of participants belonged to lower socio-economic status (53\%). Most of the individuals had education more than the secondary level. Various studies showed lower education status associated with alcohol dependence [12-13]. Seventy two percent of patients were married and living with a spouse. The same was replicated by various Indian studies [13], however, most western studies found the marital status of the patient to be being separated or divorced. The difference could be due to Indian customs and culture. Only $6 \%$ of individuals were unemployed, but most of them i.e. $44 \%$ were involved in work not requiring much education. A study done by Vignesh and others [1] found 58\% of employment in skilled work in alcohol-dependent patients.

Our study demonstrated a moderate level of dependence (40\%) in most of the participants, which is similar to previous Indian study by Singh and others [14], where $77 \%$ of participants were moderately dependent. However, a few studies included participants with severe dependence [12-13]. Both ALT (66.67\%) and AST (76.67 \%) were raised in majority of the participants, urea (96.67\%), creatinine (98.33\%), potassium (41.67\%) total bilirubin $(60 \%)$ and alkaline phosphate $(76.67 \%)$ were found to be within normal limits, and sodium (51.67\%) was found to be low in the present study. This is in accordance to previous studies which have reported high AST, ALT levels upon admission of patients [12,15]. Acute ingestion of alcohol induces a water diuresis owing to suppression of circulating vasopressin levels, predisposing patients to dehydration and hypernatremia. This suppressive effect is absent with repeated exposure or prolonged continuous exposure. In these patients, vasopressin levels increase, resulting in increased urine osmolality and decreased 
clearance of free water. As a result, hyponatremia is a common disorder that occurs in as many as $17 \%$ of patients with chronic alcohol-use disorder as reported by researchers [16-18].

However, hypokalemia was found in nearly $50 \%$ of hospitalized patients with chronic alcohol-use disorder in previous studies [16]. Researchers have observed increased serum urea and serum creatinine values in individuals with alcohol dependence syndrome [7]. Our results also found significant correlation between SADQ scores and ALT. Pradeep and others [18], also reported significant association between total SADQ scores and ALT. Gedam and others [13], however found correlation between SADQ scores and liver enzymes such as ALT AST This supports the idea that severity of dependence is associated with the liver dysfunction. The above differences in results could be attributed to heterogeneity of the population under study and tools used for measurement.

\section{CONCLUSION}

Alcohol dependence was more common in individuals with education more than secondary level. The biochemical tests were deranged in most of the patients with alcohol dependence. Certain markers can be developed based on their significant association with the dependence levels, thus, helping in early diagnosis and prevention of alcohol dependence syndrome.

\section{REFERENCES}

1. Vignesh BT, Singh AK, Mohan SK, Murthy S, Joshi A. Association between socio-demographics and alcohol dependence among individuals living in an Indian setting Glob J Health Sci. 2014;6(3):16.

2. Benegal V. India: alcohol and public health. Addiction 2005;100(8):1051-6.

3. Sharpe PC. Biochemical detection and monitoring of alcohol abuse and abstinence. Ann Clin Biochem 2001;38(6):652-64.

4. Tavakoli HR, Hull M, Okasinski LM. Review of current clinical biomarkers for the detection of alcohol dependence. Innov Clin Neurosci 2011;8(3):26.

5. Kew MC. Serum aminotransferase concentration as evidence of hepatocellular damage. Lancet 2000;355(9204):591-2.

6. Palmer BF, Clegg DJ. Electrolyte disturbances in patients with chronic alcohol-use disorder. N Engl J Med. 2017;377:1368-77.

7. Chandini P, Mathai J. Serum electrolytes levels in patients with alcohol dependence syndrome. Int J Contempor Med Res 2017;4(5):992-7.

8. Das SK, Nayak P, Vasudevan DM. Biochemical markers for alcohol consumption. Indian J Clin Biochem 2003;18(2):111-8.

9. Andó B, Rózsa S, Kurgyis E, Szkaliczki A, Demeter I, Szikszay P, Demetrovics Z, Janka Z, Álmos PZ. Direct and indirect symptom severity indicators of alcohol dependence and the personality concept of the biosocial model. Subst Use Misuse 2014;49(4):418-26.

10. Ashalata K, Kumari PK, Vijaya Babu PV, Nagamani M, Kumari KL. Serum magnesium and other electrolyte levels in chronic alcoholic patients in a tertiary mental care centre in north costal Andra Pradesh India. IOSR J Dent Med Sci 2015;14:35-7.

11. Johnson PR, Banu S, Ashok MV. Severity of alcoholism in Indian males: Correlation with age of onset and family history of alcoholism. Indian J Psychiatry 2010;52:243-9.

12. Reddy MP, Babu RS, Pathak SM, Venkateshwarlu S. The clinical and demographic profile of male patients with alcohol dependence syndrome. Indian J Psychol Med 2014;36(4):418-23.

13. Gedam SR, Dhabarde A, Patil PS, Sharma A, Kumar K, Babar V. Psychiatric Comorbidity, Severity of Dependence and Liver Enzymes Dysfunction among Alcohol Dependent Individuals: A Cross-sectional Study from Central Rural India. J Clin Diagnos Res 2019;13(4).

14. Singh A, Kumar S, Sharma CS, Dixit V, Srivastava RK, Yaduvanshi R. Other psychiatric comorbidities in male patients of alcohol dependence syndrome: a cross sectional study. Indian J Basic Appl Med Res 2016;5(2):828-38.

15. Liappas IA, Piperi C, Malitas PN, Tzavellas EO, Liappas AI, Boufidou F, et al. Correlation of liver dysfunction biological markers to the mood status of alcohol dependent individuals. Int J Psychiatry Clin Pract 2006;10(3):166-73.

16. Elisaf M, Liberopoulos E, Bairaktari E, Siamopoulos K. Hypokalaemia in alcoholic patients. Drug Alcohol Rev 2002;21:73-6.

17. Elisaf M, Kalaitzidis R. Metabolic abnormalities in alcoholic patients: focus on acid base and electrolyte disorders. J Alcohol Drug Depend 2015;3:185.

18. Pradeep RJ, Dhilip AM, Mysore A. Do SADQ and AUDIT identify independent impacts of alcohol abuse-clinical and biochemical markers respectively? Indian J Psychiatry 2015;57:278-83. 